\title{
Development of nanoemulsions containing penciclovir for herpes simplex treatment and a liquid chromatographic method to drug assessment in porcine skin layers
}

\author{
Alianise da Silva Meira*, Ana Paula Battistel, Helder Ferreira Teixeira and Nadia Maria Volpato \\ Departamento de Produção e Controle de Medicamentos, Faculdade de Farmácia, Universidade Federal do Rio Grande \\ do Sul, Porto Alegre, Brasil \\ *Corresponding author: alianise@gmail.com

\begin{abstract}
A successful formulation (penciclovir hydrogel nanoemulsion) to be used in transdermal drug delivery route to treat herpes simplex virus was developed and an analytical method to quantify penciclovir (PCV) in porcine ear skin was stablished and validated. PCV nanoemulsions were prepared by high pressure homogenization and presented spherical mean droplet size $180 \mathrm{~nm}$. The association efficiency and zeta potential were $87 \%$ and $-27 \mathrm{mV}$, respectively. The bioanalytical method developed showed specificity for the skin matrixes interferences (epidermis and dermis) and the linearity was in the range $0.1-25 \mu \mathrm{g} / \mathrm{mL}$ of drug. The mean recovery data for the three levels tested were $95.2 \%$ for the epidermis and $97.3 \%$ for the dermis and adequate results were obtained for repeatability and inter-day precision. In vitro percutaneous absorption studies with penciclovir hydrogel nanoemulsion (HN) and a market cream were conducted employing porcine skin. The PCV cumulative amounts permeated from cream and HN, 8 hours after dosing, were 2.60 and $4.15 \mu \mathrm{g} / \mathrm{cm}^{2}$, respectively, representing a quite higher flux and a much higher permeability coefficient for the developed formulation. It can be concluded that HN provide a good skin targeting effect and may be a promising carrier for topical delivery of penciclovir.
\end{abstract}

Keywords: penciclovir, nanoemulsion, cutaneous delivery studies, LC method validation, porcine skin DOI: https://doi.org/10.22456/2527-2616.103121

\section{Introduction}

Herpes simplex virus (HSV) is etiological agent behind a common skin disorder characterized by mucocutaneous lesions. Penciclovir (PCV) is an acyclic nucleoside analogue used to treat herpes virus, with efficacy against HSV type 1 and 2. Due to its poor oral bioavailability $(5-10 \%)$ and to the fact that lesions are located in the skin, topical application remains as the delivery route of choice, although numerous herpes virus systemic therapies have been developed $(1,2)$.

Even though cutaneous application is a common herpes treatment route, antivirals should be able to achieve therapeutic concentrations in basal epidermic cells, which are the development site for the virus. This is one of the main limitations of this type of treatment, largely due to poor drug penetration across the stratum corneum $(3,4)$.

The development of topical formulations containing lipidbased colloidal carriers are a promising approach to overcome this limitation and promote more effective penetration of antiviral drugs through the skin and thus to reduce the frequency of application $(4,5)$.

Nanoemulsions have large interfacial area and small droplet size, which are attractive characteristics for applications in pharmaceutical and cosmetics industries $(6,7)$. One of the nanoemulsions advantages is the large production scale possibility by high pressure homogenization. This technology can be summarized in three steps: (1) formation of preemulsion; (2) pre-dispersion homogenized by the highpressure homogenizer at a low pressure several times, and (3) homogenization at a high pressure for a sufficient number of cycles so that nanoemulsions achieve the desired size $(8,9)$. Moreover, this technique is fast, simple and reliable for nanoemulsions preparation, apart from the capacity to incorporate drugs that are difficult to solubilize in aqueous and organic solvents, such as PCV $(\log P-1.62)(9,10)$. In spite of the fact that many anti-herpes drugs have been incorporated in nanoemulsions, penciclovir has not been evaluated $(11,12)$.

To evaluate the performance of new semisolid formulations with respect to the release, penetration and absorption of drugs in and/or through the skin the in vitro percutaneous absorption studies are conducted employing Franz diffusion cell and a cutaneous tissue model (13).

Drug retention or permeation in the skin after the in vitro percutaneous absorption studies can be evaluated based on analytical quantification method previously validated, so as to ensure and to demonstrate the quality of the results, since the lack of reliable analytical data may lead to misleading decisions (14).

The aim of this study was to develop nanoemulsions containing PCV for herpes simplex treatment and develop a liquid chromatographic method applied to drug quantification in porcine skin layers as well as to conduct in vitro percutaneous absorption studies for the formulation developed compared to a commercial cream.

\section{Materials and Methods}

PCV was obtained from EMS (São Paulo, Brazil). Egglecithin (Lipoid E-80®) and medium chain triglycerides (MCT) were obtained from Lipoid GmbH (Ludwigshafen, Germany). Poloxamer 188 was obtained from Vetec (Rio de Janeiro, Brazil) and carbomer 940 was purchased from Sigma (São Paulo, Brazil). PCV commercial formulation at concentration $1 \%(\mathrm{w} / \mathrm{w})$ was obtained from a local pharmacy. All the others reagents used in this work were HPLC grade. Acetonitrile and methanol were purchased from Tedia (Fairfield, USA) and the phosphoric acid from Merck (Darmstadt, Germany). Purified water was obtained by a Millipore ${ }^{\circledR}$ Direct-Q 3UV (Molsheim, France). The porcine ears were obtained from Ouro do Sul slaughterhouse (Harmonia, Brazil). 


\section{LC method}

The LC apparatus consisted of a Shimadzu LC-10A system (Kyoto, Japan) equipped with a model LC-20AT pump, a SPD-20AV UV-VIS variable wavelength detector (set at 251 $\mathrm{nm}$ ), a degasser DGU-20A5, a CBM-20A system controller, and SIL-20A injection valve with a $100 \mu \mathrm{L}$ loop. PCV was analyzed using a Phenomenex C8 column $(5 \mu \mathrm{m}, 250 \mathrm{~mm}$ x $4.6 \mathrm{~mm}$ i.d.) and Phenomenex C8 security guard (4 x 3.0 $\mathrm{mm})$. The selected phase consisted of a methanol:water mixture $(7: 93 \mathrm{w} / \mathrm{w})$ in isocratic mode. Flow rate of $1 \mathrm{~mL} / \mathrm{min}$ and an injection volume of $60 \mu \mathrm{L}$ at room temperature were employed.

\section{Skin Preparation}

The porcine ears were obtained from a local slaughterhouse (16-18 hours after slaughter). The pig ears were washed with water and the skin of central region from the outer side of the ear was excised using a scalpel. After that, they were packed in polyvinyl chloride film and aluminum foil, stored at $-20^{\circ} \mathrm{C}$ and used within one month (15).

\section{Penciclovir Extraction Procedure}

For extraction procedure development, pig ear skin samples were sectioned in slices of $1.5 \mathrm{~cm}$ of diameter (equivalent to contact area with the formulation in the Franz cells). The skin layers were separated according to the following procedure: the skin circles were submerged in water at $60{ }^{\circ} \mathrm{C}$ for 45 seconds, then epidermis and dermis layers were separated with a scalpel, cut into small pieces and placed in different tubes. Next, known amounts of the penciclovir (PCV) solution were poured over the tissues. After impregnation time and solvent evaporation (around 4 hours), $4 \mathrm{~mL}$ of extractor solvent - water or $\mathrm{HCl} 0.1 \mathrm{M}$ - were added, resulting in theoretical concentration final of 12.5 and $25 \mu \mathrm{g} / \mathrm{mL}$. Different extraction times (20, 30 and 45 minutes) by ultrasound were tested. After that, the solution was filtered into vials and analyzed by HPLC.

\section{LC Method Validation}

The PCV quantification method in porcine skin samples was validated according to ICH guideline (16). For specificity evaluation, the chromatograms without and with PCV addition at epidermis and dermis obtained after extraction procedure were compared. Similarly, the specificity method for the formulations was assessed by comparing the chromatograms obtained from the products containing PCV and from blank formulation (placebo).

Linearity was evaluated in the range $0.1-25 \mu \mathrm{g} / \mathrm{mL}$ by seven drug concentrations prepared in three different days. The final dilution of each volumetric flask was done with water spiked with porcine skin (epidermis and dermis) in the same proportion obtained during the extraction procedure (round skin of $1.5 \mathrm{~cm}$ diameter for each $8 \mathrm{~mL}$ of diluent). Linear regression was calculated by the least square regression method, and ANOVA was done to assess compliance to the linear model. The quantification and detection limits (LOQ and LOD, respectively) were formerly estimated based on standard curves (linearity). For the real quantification limits, three solutions $(0.1,0.05$ and $0.03 \mu \mathrm{g} / \mathrm{mL})$ were analyzed for precision and accuracy based on six injections for each concentration.

The accuracy of the method was evaluated by PCV added to blank skin layer and its amount recovery. Three different final concentrations (low, intermediate and high, 0.5, 12.5 and $25 \mu \mathrm{g} / \mathrm{mL}$, respectively) for each layer (epidermis and dermis) were prepared in triplicate for two consecutive days, resulting in six measurements for each level and each layer. Intra-day and inter-day precision were evaluated by RSD from accuracy results replicates. Additionally, carry-over was checked through three blank samples injection after a high level $(25 \mu \mathrm{g} / \mathrm{mL})$ sample analyze.

\section{Preparation of Penciclovir-loaded nanoemulsions}

Nanoemulsions $\mathrm{O} / \mathrm{W}$ containing PCV were prepared using the high-pressure homogenization (HPH) method. The nanoemulsions oily phase was composed by MCT, egglecithin and PCV; aqueous phase was composed by poloxamer 188 and water. The phases were heated individually at $60{ }^{\circ} \mathrm{C}$, to complete solubilization of the components. After that, the phases were mixed and magnetically stirred for 5 minutes to form a pre-emulsion. Then, the pre-emulsion was submitted to HPH equipment at 10,000 psi in 6 cycles (17). Blank formulations were prepared under the same conditions in absence of PCV and used as control. For hydrogels containing PCV-loaded nanoemulsions, carbomer 940 was dispersed in water and stirred at room temperature using a magnetic stirrer. After this, the mixture was neutralized to $\mathrm{pH}$ 6.0-7.0 with triethanolamine, to form the gel. Finally, $0.5 \mathrm{~g}$ of the carbomer gel was mixed with $4.5 \mathrm{~g}$ nanoemulsion with continuous stirring. The final composition (\%,w/w) of nanoemulsions and hydrogel nanoemulsions obtained in presence or absence of poloxamer 188 are presented in Table 1.

Table 1. Final composition (\%, w/w) of PCV-loaded nanoemulsions incorporated into hydrogels.

\begin{tabular}{lcc}
\hline & F1 & F 2 \\
\hline PCV & 0.1 & 0.1 \\
MCT & 8 & 8 \\
Egg-lecithin & 2 & 2 \\
Poloxamer 188 & - & 1 \\
Water q.s.p & 100 & 100 \\
\hline Carbomer 940 & 0.5 & 0.5 \\
Thrietanolamine & 0.2 & 0.2 \\
\hline
\end{tabular}

MCT: medium chain triglycerides

\section{Physicochemical characterization of nanoemulsions and hydrogels}

The nanoemulsions were characterized by mean droplet size (MDS), polydispersity index (PDI), zeta potential (ZP) and $\mathrm{pH}$. MDS, PDI and ZP were determined by photon correlation spectroscopy (PCS) and electrophoretic mobility, respectively $(3000 \mathrm{HS}$ Zetasizer, Malvern Instruments, England). All the samples were diluted to 0.4:200 (v/v) with $1 \mathrm{mM} \mathrm{NaCl}$ before measurement. Also, $\mathrm{pH}$ was determined 
directly in the bulk for each formulation using a pHmeter Digimed 20, and viscosity was determined using an Ostwald capillary viscometer at $20{ }^{\circ} \mathrm{C}$. The nanoemulsions morphology was assessed by transmission electron microscopy (TEM).

After incorporation of nanoemulsions into hydrogels, the morphological examination was also performed using TEM. Further, the rheological characterization of the hydrogel formulations were carried out using a Brookfield LVLD-II+ rotational viscometer at room temperature $\left(25 \pm 1^{\circ} \mathrm{C}\right)$.

\section{Penciclovir content and association efficiency (AE)}

For PCV content determination, $500 \mu \mathrm{L}$ of nanoemulsions was appropriately dissolved and diluted in methanol. An aliquot from the solutions was diluted in water, filtered and analyzed by liquid chromatography. For association efficiency, $500 \mu \mathrm{L}$ of PCV-loaded nanoemulsions was placed into an ultrafiltration device (Amicon® Ultra-4, Millipore, Ireland) with a $30 \mathrm{kDa}$ molecular weight cut-off which was centrifuged at $10,000 \mathrm{rpm}$ for $10 \mathrm{~min}$ at $25^{\circ} \mathrm{C}$. The free drug amount in the external aqueous phase after separation from nanoemulsions was determined by liquid chromatography. The incorporated drug amount was calculated as result of the initial drug minus free drug. The association efficiency (AE) was calculated according to the following equation:

$A E(\%)=\left[\left(W_{\text {initial drug }}-W_{\text {free drug }}\right) / W_{\text {initial drug }}\right] \times 100 \%$; where " $W_{\text {initial drug" }}$ is the amount of drug used for the assay and the " $W_{\text {free drug }}$ " is the amount of free drug quantified in the aqueous phase after isolation of the emulsion droplets.

\section{In vitro skin percutaneous absorption studies}

The suitability and the new formulation evaluation were performed in percutaneous absorption studies using a Franz type diffusion cell - area of $1.77 \mathrm{~cm}^{2}$ with $7 \mathrm{~mL}$ of volume in the receptor compartment (Hanson Research, Chatsworth, USA) and porcine skin as membrane. The conditions employed for studies were: buffer phosphate $\mathrm{pH} 7.4$ as receptor solution (PCV solubility is $1.3 \mathrm{mg} / \mathrm{mL}$ in physiological saline) at $32{ }^{\circ} \mathrm{C}$ and stirring at $500 \mathrm{rpm}$.

The full-thickness porcine skin samples were mounted carefully on Franz-type diffusion cells with the stratum corneum side up the donor compartment and about $300 \mathrm{mg}$ of test formulation (PCV hydrogel nanoemulsions or PCV market formulation) was placed on the skin surface in the donor compartment. The total experiment time was 8 hours and at given time intervals (1, 2, 4, 6 and 8 hours) receptor fluid aliquots of $1 \mathrm{~mL}$ were collected and the same volume were replaced. After 8 hours contact, the skin was removed from diffusion cell, the excess of formulation was carefully removed and the skin surface was gently cleaned with a wet swab. Immediately after, skin layers were separated by scapel, cut, weighed and submitted to the extraction procedure previously described. Only the skin area just in contact with the products was employed for extraction.

The permeation flux $J\left(\mu \mathrm{g} / \mathrm{cm}^{2} / \mathrm{h}\right)$ was determined employing the simplest approach by fitting the linear portion of the experimental curves for PCV permeated versus time and calculating the slope. To determine the permeability coefficient $P(\mathrm{~cm} / \mathrm{h})$, the flux $J$ was divided by the PCV concentration in the donor compartment $C_{d o n o r}\left(\mu \mathrm{g} / \mathrm{cm}^{3}\right)$ to normalize the different drug concentration of the products at starting time (18).

\section{Results and Discussion}

\section{LC method and Penciclovir extraction procedure}

For PCV extraction procedure development from skin layers, two solvents in which the drug is soluble - water and hydrochloric acid - and different times of extraction (20, 30 and 45 minutes) were tested. The results showed better, considering the recovery index and the polar similarity with the mobile phase of the chromatographic system, when using water as extractor solvent and 45 minutes was the time of extraction (data not shown). The analytical conditions employed allowed the adequate resolution of the PCV signal from the epidermis and dermis components, demonstrating specificity by the absence of interfering from biological matrix for each layer in the PCV retention time (approximately 10 minutes) (Figure 1).

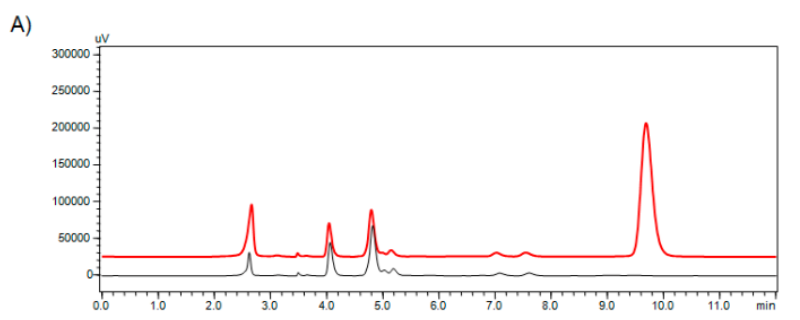

B)

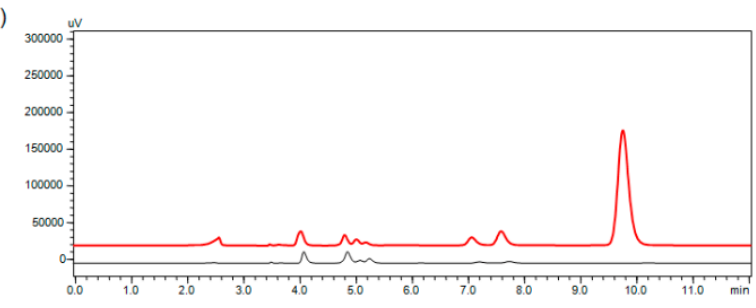

Figure 1. Specificity of the chromatographic system for PCV analysis in skin layers. A) Epidermis; B) Dermis. Black line is the blank of the skin layer; red is the skin layer with PCV $25 \mu \mathrm{g} / \mathrm{mL}$. $\mathrm{PCV}$ retention time is $9.8 \mathrm{~min}$.

Satisfactory linearity could be obtained in the evaluated concentration range on each day during the experiment $\left(\mathrm{r}^{2}=\right.$ 0.9999), and ANOVA showed non-significant linear deviation ( $p>0.05)$. LOQ and LOD were 0.1 and $0.03 \mu \mathrm{g} / \mathrm{mL}$, respectively, when determined by estimation from the linear equations. When evaluated in the experimental assay, the concentrations tested $(0.1,0.05$ and $0.03 \mu \mathrm{g} / \mathrm{mL})$ presented $0.3 \%$ and $113.2 \% ; 4.2 \%$ and $89.4 \% ; 4.5 \%$ and $64.4 \%$, for RSD and accuracy, respectively, demonstrating that LOQ is minor the expected value, namely, $0.05 \mu \mathrm{g} / \mathrm{mL}$. The accuracy of the analytical method is presented in terms of percentage recoveries of PCV added to biological matrix. Table 2 shows the mean data obtained for PCV recovery from skin layers (epidermis and dermis) and the values of relative standard deviation (RSD) for repeatability (intra-day) and intermediate precision for each level of PCV added to the matrixes. 
Table 2. Accuracy and precision for PCV analysis in the skin layers by LC method

\begin{tabular}{|c|c|c|c|c|c|c|}
\hline \multirow[b]{2}{*}{$\begin{array}{l}\text { Skin } \\
\text { layers }\end{array}$} & \multirow[b]{2}{*}{$\begin{array}{l}\text { PCV } \\
\text { Level }\end{array}$} & \multicolumn{2}{|c|}{ Day 1} & \multicolumn{2}{|c|}{ Day 2} & \multirow[b]{2}{*}{$\begin{array}{c}\text { Inter } \\
\text { Day } \\
\text { RSD }\end{array}$} \\
\hline & & $\begin{array}{l}\mathrm{AR} \\
(\%)\end{array}$ & $\begin{array}{r}\text { Intra } \\
\text { Day } \\
\text { RSD }\end{array}$ & $\begin{array}{l}\text { AR } \\
(\%)\end{array}$ & $\begin{array}{r}\text { Intra } \\
\text { Day } \\
\text { RSD }\end{array}$ & \\
\hline \multirow{3}{*}{ E } & $\mathrm{L}$ & 93.47 & 12.8 & 96.10 & 0.86 & 7.97 \\
\hline & I & 95.84 & 6.74 & 92.99 & 7.08 & 6.40 \\
\hline & $\mathrm{H}$ & 98.99 & 1.43 & 93.99 & 5.69 & 4.61 \\
\hline \multirow{3}{*}{$\mathrm{D}$} & $\mathrm{L}$ & 104.44 & 5.34 & 101.5 & 3.15 & 4.50 \\
\hline & I & 89.70 & 8.47 & 89.90 & 1.96 & 5.57 \\
\hline & $\mathrm{H}$ & 96.74 & 4.16 & 98.33 & 0.82 & 5.03 \\
\hline
\end{tabular}

$\mathrm{E}=$ epidermis; $\mathrm{D}=$ dermis; $\mathrm{L}=$ low; I= Intermediate; $\mathrm{H}=\mathrm{High} ; \mathrm{AR}=$ Average recovery.

The results are in accordance to the literature recommendations of overall recovery in the range of $85-$ $115 \%$ for bioanalytical methods (16). The RSD values were also considered acceptable for a bioanalytical method $(<15 \%)$, indicating the suitability of extraction process and analysis for quantification of PCV in the main skin layers. To ensure the accuracy (recovery) of the analytical method regarding skin sample preparation, a second extraction procedure was conducted with the same skin layers immediately after the first extraction. No more than $10 \%$ of the amount determined in the first extraction was found for both layers, demonstrating that the procedure was suitable for extraction of PCV retained in porcine skin layers (data not shown). Carry-over from the analytes was not observed at all.

\section{Formulation study}

Initially, six types of nanoemulsions were prepared with increasing PCV amounts. The formulations were obtained in the absence or presence of a non-ionic surfactant, poloxamer 188. Firstly, 0.1\% PCV nanoemulsions (F1 and F2) were prepared. In attempt to reach the PCV concentration in the commercial formulation (1\%), nanoemulsions $0.5 \%$ and $1 \%$ were also prepared. However, these formulations proved to be unstable few hours after preparation, precipitating crystals overnight, even with the increase in additional surfactant $(9,19)$. Therefore, F1 and F2 nanoemulsions were selected for further characterization studies. Table 3 exhibits the main physicochemical properties of blank (BF) and PCV-loaded nanoemulsions $(\mathrm{F})$.

Table 3. Physicochemical properties of blank (BF) and PCV-loaded nanoemulsions $(\mathrm{F})$.

\begin{tabular}{lccccc}
\hline F & $\begin{array}{c}\text { Size } \\
(\mathbf{n m})\end{array}$ & PDI & $\begin{array}{c}\zeta \\
(\mathbf{m V})\end{array}$ & $\begin{array}{c}\mathbf{V} \\
(\mathbf{c P})\end{array}$ & $\mathbf{p H}$ \\
\hline F1 & 212.6 & 0.163 & -26.9 & 0.83 & 6.39 \\
& $( \pm 1.79)$ & $( \pm 0.01)$ & $( \pm 0.90)$ & $( \pm 0.004)$ & \\
BF1 & 219.7 & 0.148 & -27.1 & 0.82 & 6.99 \\
& $( \pm 2.65)$ & $( \pm 0.02)$ & $( \pm 2.34)$ & $( \pm 0.14)$ & \\
F2 & 186.8 & 0.102 & -27.7 & 1.13 & 5.89 \\
& $( \pm 3.34)$ & $( \pm 0.01)$ & $( \pm 0.84)$ & $( \pm 0.12)$ & \\
BF2 & 190.3 & 0.078 & -26.1 & 1.11 & 5.62 \\
& $( \pm 4.25)$ & $( \pm 0.02)$ & $( \pm 0.69)$ & $( \pm 0.16)$ & \\
\hline $1 \cdot$ & nanoemulsion & without poloxamer; 2. & nanoemulsion with
\end{tabular}

1: nanoemulsion without poloxamer; 2: nanoemulsion with poloxamer; PDI: polydispersity index; V: Viscosity.
Regardless of poloxamer 188 presence, mean droplet size was in the $180-220 \mathrm{~nm}$ range. F2 presented smaller size and $\mathrm{pH}$ than F1, which can be attributed to the presence of an additional surfactant, apart from egg lecithin. Adequate polydispersity index (lower than 0.17) was observed, showing a low deviation from the average size, where values up to 0.25 have been acceptable (20). The nanoemulsions presented $\zeta$-potential about $-27 \mathrm{mV}$, indicating good stability of the formulations (17). Negative $\zeta$-potential values are attributed to the presence of negatively charged phospholipids of egg lecithin in the final $\mathrm{pH}$ of the formulations. Moreover, the PCV incorporation into the nanoemulsions did not change $\zeta$-potential values.

The formulations exhibited low viscosity (F1: $0.82 \mathrm{cP}$; F2: $1.13 \mathrm{cP}$ ), though $\mathrm{F} 2$ formulation viscosity was significantly higher $(\mathrm{p}<0.05)$ when compared to the value observed for F1 (Table 3). Therefore, to favor nanoemulsions application on the skin is recommended that viscosity had been adjusted to reach a semisolid form. Thence, the nanoemulsions were incorporated into carbomer hydrogel. The final formulation (PCV hydrogel nanoemulsion - HN) shown to be nonNewtonians fluids, since viscosity of fluid varies nonlinearly with shear rate without thixotropic behavior (data not shown) (21). Regarding the morphology, F2 nanoemulsions photomicrographs revealed spherical oil nanodroplets slightly larger than $150 \mathrm{~nm}$ in diameter, in accordance with the results obtained by dynamic light scattering (Figure 2).
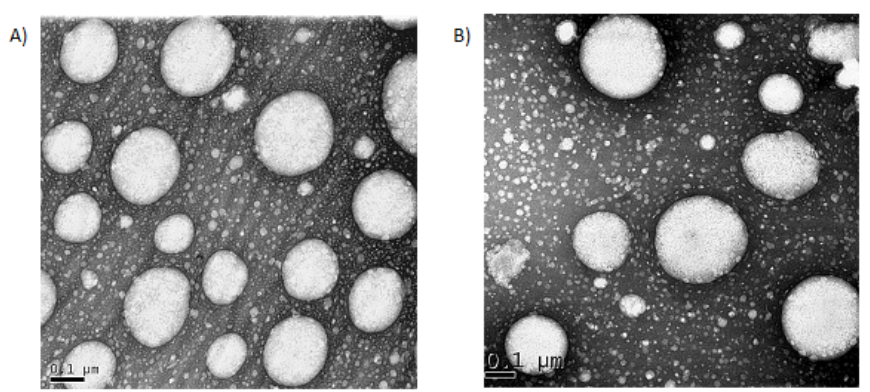

Figure 2. Photomicrographs of PCV loaded-nanoemulsions obtained from transmission electronic microscopy for F2 formulation before (A) and after (B) incorporation in hydrogel.

Figure 2B shows individual oil droplets, despite their dispersion in the polymer network, exhibiting a droplet size quite similar to those detected before gel thickening, in turn indicating, under conditions applied in this study, that the nanoemulsions structure before being obtained as a semisolid preparation was in fact maintained.

\section{Penciclovir content and association efficiency (AE)}

PCV content was determined by liquid chromatography and the results for nanoemulsions and HN are presented in Table 4. 
Table 4. PCV content $(\%)^{*}$ in nanoemulsions and after incorporation in hydrogels $(\mathrm{HN})$, at day 1 and after 30 days of storage at $4^{\circ} \mathrm{C}$.

\begin{tabular}{ccccc}
\hline & \multicolumn{2}{c}{ Nanoemulsions } & \multicolumn{2}{c}{ HN } \\
\hline & Initial & 30 days & Initial & 30 days \\
\hline F1 & 89.88 & 84.28 & 88.57 & 82.45 \\
& $( \pm 0.65)$ & $( \pm 2.62)$ & $( \pm 2.54)$ & $( \pm 3.67)$ \\
F2 & 95.83 & 92.39 & 94.27 & 91.27 \\
& $( \pm 2.70)$ & $( \pm 3.96)$ & $( \pm 1.69)$ & $( \pm 0.28)$ \\
\hline
\end{tabular}

*For a PCV theoretical concentration of $1 \mathrm{mg} / \mathrm{mL}$.

The F2 formulation presented quite higher PCV content than F1. This result was also observed for association efficiency, where $\mathrm{F} 2$ showed around $87 \%$ of the drug loaded into internal phase of the emulsion, while F1 presented around $75 \%$ of association. This result for F2 can be due to the presence of poloxamer 188 as stabilizer in addition to egg-lecithin (14). Therefore, F2 nanoemulsion was selected for further studies of cutaneous delivery. The commercial cream was also checked regarding PCV content and it was in accordance with the labeled dose.

\section{In vitro skin percutaneous absorption studies}

The PCV cumulative amount determined in the skin layers for each formulation is shown in Figure 3.

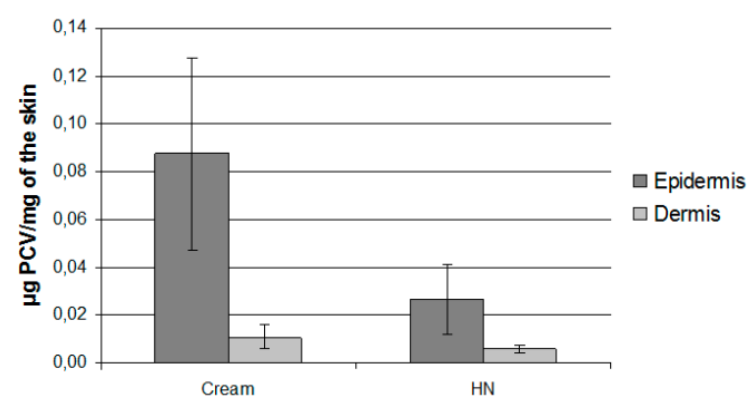

Figure 3. Amount of extracted PCV from the skin layers expressed as $\mu \mathrm{g} / \mathrm{mg}$. *HN: hydrogel nanoemulsion.

For epidermis and dermis, the PCV amounts retained, respectively, were 0.09 and $0.01 \mu \mathrm{g} / \mathrm{mg}$ for the cream and 0.03 and $0.006 \mu \mathrm{g} / \mathrm{mg}$ for the F2-HN. The PCV percutaneous permeation profiles through porcine skin are shown in Figure 4.

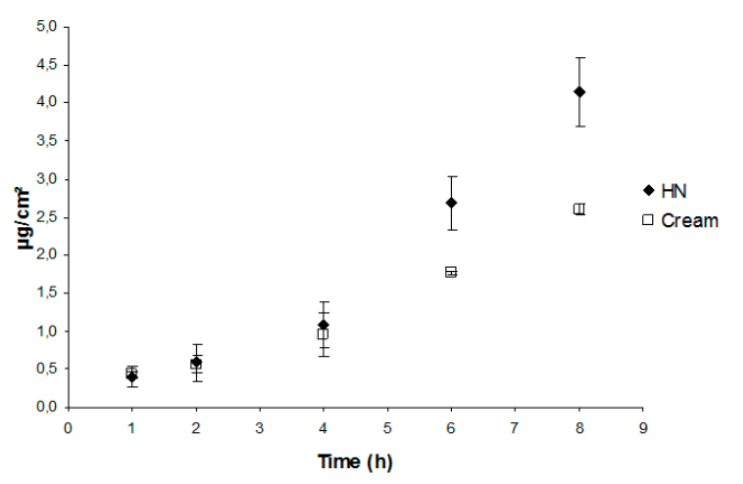

Figure 4. Percutaneous permeation profiles of penciclovir from the hydrogel nanoemulsions and commercial cream through pig ear skin at time intervals 1, 2, 4, 6 and 8 hours.
The drug flux $(J)$ from the cream was $0.41 \mu \mathrm{g} / \mathrm{cm}^{2} / \mathrm{h}$ and from F2-HN was $0.77 \mu \mathrm{g} / \mathrm{cm}^{2} / \mathrm{h}$ leading to a permeability coefficient $(P)$ of $4.10^{-5} \mathrm{~cm} / \mathrm{h}$ for the cream and $76.10^{-5} \mathrm{~cm} / \mathrm{h}$ for the developed formulation. The PCV cumulative amounts permeated from cream and F2-HN, eight hours after dosing were 2.60 and $4.15 \mu \mathrm{g} / \mathrm{cm}^{2}$, respectively. These results were surprising as PCV concentration in cream is ten folds higher than in F2-HN. This observation might support the hypothesis that F2-HN has the tendency to have a higher passage through stratum corneum, as revealed by higher permeability coefficient, which makes it an attractive choice as the carrier for topical delivery of PCV.

\section{Conclusions}

The analytical method developed showed good specificity, accuracy and precision to measure PCV into porcine skin samples (epidermis and dermis). The method's applicability to the work was proved through the evaluation of PCV skin retention and permeation from two formulations, commercial cream and hydrogel nanoemulsion developed. Furthermore, despite the minor PCV concentration in the new product, a higher skin permeability from hydrogel nanoemulsion was detected for the drug.

\section{Acknowledgments}

The authors are grateful to $\mathrm{CNPq}$ (Brazil), for their support.

\section{Conflict of interest}

The authors declare no conflicts of interest.

\section{References}

1. Lin L, Chen XS, Cui PG, Wang JB, Guo ZP, Lu NZ. Topical application of penciclovir cream for the treatment of herpes simplex facialis / labialis : a controlled trial BACKGROUND : 2002;67-72.

2. Yu A, Guo C, Zhou Y, Cao F, Zhu W, Sun M, et al. International Immunopharmacology Skin irritation and the inhibition effect on HSV-1 in vivo of penciclovirloaded microemulsion. Int Immunopharmacol [Internet]. 2010;10(10):1305-9. Available from: http://dx.doi.org/10.1016/j.intimp.2010.07.015

3. Hasler-nguyen N, Shelton D, Ponard G, Bader M, Schaffrik M, Mallefet $P$. penciclovir $1 \%$ cream and acyclovir $5 \%$ cream used to treat herpes simplex virus infection. 2009;10:1-10.

4. Lv Q, Yu A, Xi Y, Li H, Song Z, Cui J, et al. Development and evaluation of penciclovir-loaded solid lipid nanoparticles for topical delivery. 2009;372:191-8.

5. Lopes CM, Silva J, Real Oliveira MECD, Lúcio M. Lipidbased colloidal carriers for topical application of antiviral drugs. In: Design of Nanostructures for Versatile Therapeutic Applications. 2018. p. 565-622.

6. Sadurní N, Solans C, Azemar N, García-Celma MJ. Studies on the formation of O/W nano-emulsions, by lowenergy emulsification methods, suitable for pharmaceutical applications. Eur $\mathrm{J}$ Pharm Sci. 
2005;26(5):438-45.

7. Solans C, Izquierdo P, Nolla J, Azemar N. Nanoemulsions. 2005;10:102-10.

8. Almeida ME, Teixeira HF, Koester LS. Preparação de Emulsões Submicrométricas : Aspectos Teóricos sobre os Métodos Empregados na Atualidade. 2008;27(5):780-8.

9. Pu X, Sun J, Li M, He Z. Formulation of Nanosuspensions as a New Approach for the Delivery of Poorly Soluble Drugs. 2009;(59):417-27.

10. Mehnert W, Mäder K. Solid lipid nanoparticles is Production, characterization and applications. Adv Drug Deliv Rev [Internet]. 2012;64:83-101. Available from: http://dx.doi.org/10.1016/j.addr.2012.09.021

11. Jensen LB, Petersson K, Nielsen HM. European Journal of Pharmaceutics and Biopharmaceutics In vitro penetration properties of solid lipid nanoparticles in intact and barrier-impaired skin. Eur J Pharm Biopharm [Internet]. 2011;79(1):68-75. Available from: http://dx.doi.org/10.1016/j.ejpb.2011.05.012

12. Trimaille T, Chaix C, Delair T, Pichot C, Teixeira H, Dubernet $\mathrm{C}$, et al. Interfacial deposition of functionalized copolymers onto nanoemulsions produced by the solvent displacement method. Colloid Polym Sci. 2001;279(8):784-92.

13. Schäfer-korting $M$, Mehnert $W$, Korting H. Lipid nanoparticles for improved topical application of drugs for skin diseases is. 2007;59:427-43.

14. Jardim MRCBGBCHCICSF. Revisão - Validação de métodos cromatográficos e eletroforéticos. Quim Nova. 2004;27(5):771-80.

15. Barth AB, Pereira RL, Vargas BA De, Volpato NM. A simple and rapid method to assess butena fi ne hydrochloride in skin samples and a comparative cutaneous retention study of two marketed formulations. 2010;(October).

16. Fda CC. Guidance for Industry Q2B Validation of Analytical Procedures: Methodology. 1800;(November 1996).

17. Mehnert W, Mader K. Solid lipid nanoparticles Production , characterization and applications. 2001;47:165-96.

18. Henning A, Schaefer UF, Neumann D. Potential pitfalls in skin permeation experiments: Influence of experimental factors and subsequent data evaluation. Eur J Pharm Biopharm [Internet]. 2009;72(2):324-31. Available from: http://dx.doi.org/10.1016/j.ejpb.2008.07.016

19. Patravale VB, Date AA, Kulkarni RM. Nanosuspensions : a promising drug delivery strategy. 2004;(1992):827-40.

20. Müller RH, Schmidt S, Buttle I, Akkar A, Schmitt J, Brömer S. SolEmuls ${ }^{\circledR}$ - Novel technology for the formulation of i.v. emulsions with poorly soluble drugs. Int J Pharm. 2004;269(2):293-302.

21. Lee CH, Moturi V, Lee Y. Thixotropic property in pharmaceutical formulations. J Control Release [Internet]. 2009;136(2):88-98. Available from: http://dx.doi.org/10.1016/j.jconrel.2009.02.013 